\title{
Análise Energética e Exergética para o Ciclo Dual em Motores de Alta Rotação
}

\author{
Antônio Chicharo Prata Lisboa ${ }^{1}$, Claudia Mazza Dias ${ }^{1}$ \& Julio Henrique Lopes de \\ Almeida ${ }^{2}$
}

(1) Universidade Federal Rural do Rio de Janeiro, Instituto de Ciências Exatas, Programa de Pós-Graduação em Modelagem Matemática e Computacional, Rodovia BR-465, Seropédica 23897-000, Rio de Janeiro, Brasil. E-mail: antonio.chicharo@gmail.com, mazzaclaudia@gmail.com

(2) Universidade do Estado do Rio de Janeiro, Faculdade de Engenharia, Programa de Pós-Graduação em Engenharia Eletrônica, Rua São Francisco Xavier 524, Maracanã 20550-900, Rio de Janeiro, Brasil. Email: jh.juliohenrique@gmail.com

Lisboa A.C.P., Dias C.M. \& Almeida J.H.L. (2018) Análise Energética e Exergética para o Ciclo Dual em Motores de Alta Rotação. Pesquisa e Ensino em Ciências Exatas e da Natureza, 2(edição especial): 15-29. http://dx.doi.org/10.29215/pecen.v2i2.1037

Editor acadêmico: Gustavo B. Alvarez. Recebido: 02 Junho 2018. Aceito: 12 Setembro 2018. Publicado: 26 Novembro 2018.

Resumo: Este trabalho tem como propósito fazer uma análise do Ciclo Dual, adequado a motores de alta rotação, com o objetivo de obter suas eficiências Energética e Exergética, bem como quantificar a destruição de exergia nos processos. Os resultados são obtidos através de estudos paramétricos que permitem a avaliação do comportamento dos parâmetros de interesse. Todos os cálculos são desenvolvidos em um código aberto escrito em Python ${ }^{\circledR}$ com o suporte das bibliotecas numpye scipy.

Palavras chave: Ciclo Dual, Modelagem Computacional, Eficiência Exergética.

\section{Energy and Exergy Analysis of the Dual Cycle in High Rotation Engines}

Abstract: This work has the purpose of performing the Dual Cycle analysis, suitable for high rotation engines, with the objective of obtaining its Energetic and Exergetic efficiencies, as well as quantifying the destruction of exergy in the processes. The results are obtained through parametric studies, which allow the evaluation of the behavior of parameters of interest. The calculations are developed in an open code written in Python ${ }^{\circledR}$ with the support of libraries numpy and scipy.

Key words: Dual Cycle, Computational Modeling, Exergetic Efficiency.

\section{Introdução}

O funcionamento de um motor real é ditado pela combinação de características construtivas como: a geometria do motor, enquadramento do comando de válvulas, etc.; características do combustível como: a velocidade de queima, condição do comburente, taxa de liberação de energia, etc.; e ainda, das características de controle como: a forma de injeção, ponto de ignição/injeção, comando de válvula variável, etc. Como os aspectos construtivos e de combustível são fixos, fica a cargo das características de controle garantir o funcionamento eficiente do motor. Porém, sua atuação é limitada devido a combinação dos aspectos fixos com a ampla faixa de rotação exigidas. Portanto, na condição de operação real, onde tem-se alta rotação do motor, seja de ignição por centelha (Ciclo Otto) ou de ignição por compressão (Ciclo Diesel), percebe-se no diagrama Pressão (P) - Velocidade (V), P-V, que ocorre o aumento da pressão no 
início da combustão, representada por uma isocórica, ou seja, à pressão constante. Logo depois observa-se um patamar de pressão que pode ser representado por uma curva isobárica, ou seja, á volume constante (Figuras 1-2), pois o pistão se desloca rapidamente nesta situação. Como consequência, a combustão tem menos tempo para acontecer com pequenas variações da posição do pistão, o que pode ser considerado eficiente, e objetivo do controle. Deste modo, o patamar de pressão, no diagrama $\mathrm{P}-\mathrm{V}$, aumenta, já que parte da combustão ocorre enquanto o pistão está se deslocando no sentido do aumento do volume. Assim, o aumento da pressão proveniente da combustão é anulado (Heywood 2011). Como na prática tanto os motores de ignição por centelha quanto os de ignição por compressão, em alta rotação, não possuem o fornecimento de calor totalmente de forma isocórica ou isobárica, respectivamente, a modelagem mais adequada para o problema consiste em considerar um pico de pressão a volume constante e um patamar de pressão constante. Esse modelo é chamado de Ciclo Dual, e ainda traz a vantagem de obter como casos particulares os chamados Ciclos Otto e Diesel (Ebrahimi 2010). Boa parte dos livros de termodinâmica para engenharia trazem como modelos para motores de combustão interna alternativos apenas o Ciclo Otto ou o Ciclo Diesel, e deste modo não conseguem representar precisamente o funcionamento real do motor. Assim, o presente trabalho, tem como alvo o Ciclo Dual, permitindo uma modelagem mais precisa do processo de aporte de calor. Para analisar a eficiência desses motores, o trabalho propõe a análise energética e a análise exergética, permitindo desta forma a obtenção dos parâmetros da modelagem através de estudos paramétricos.

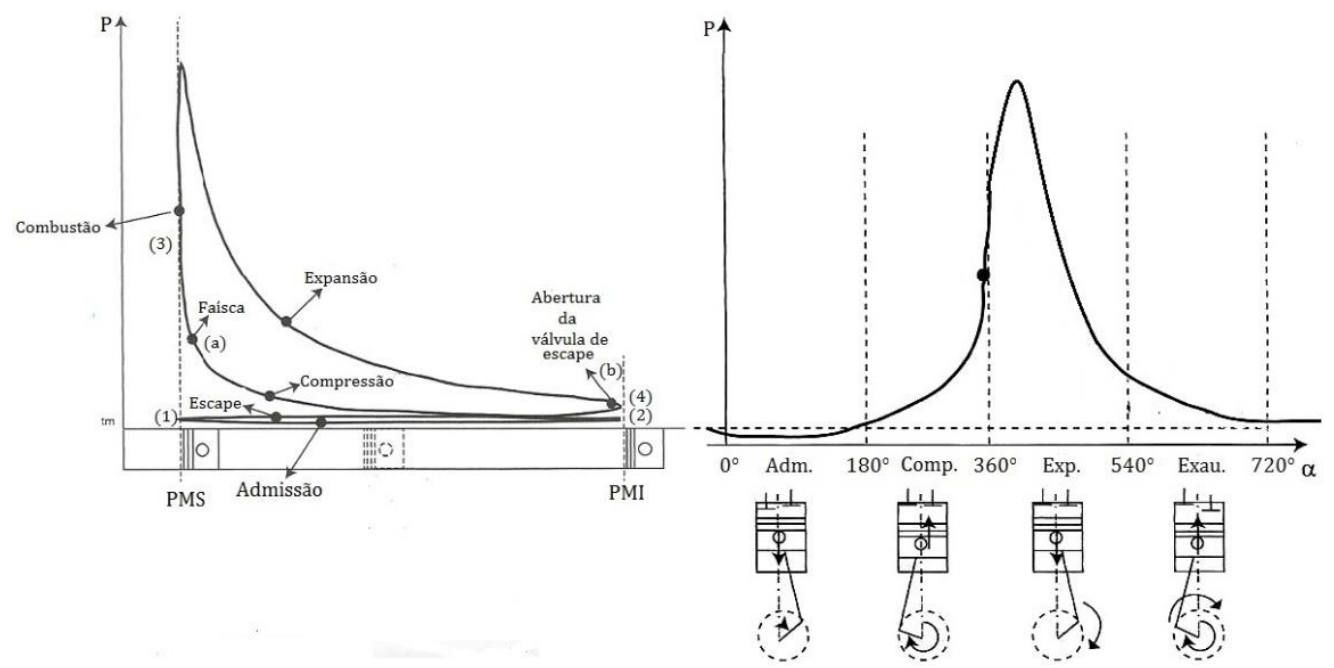

Figura 1. Diagrama P-V em relação ao movimento de translação do pistão e diagrama P-V em relação ao ângulo $(\alpha)$ do virabrequim do motor a combustão por centelha (Brunetti 2012: 86).

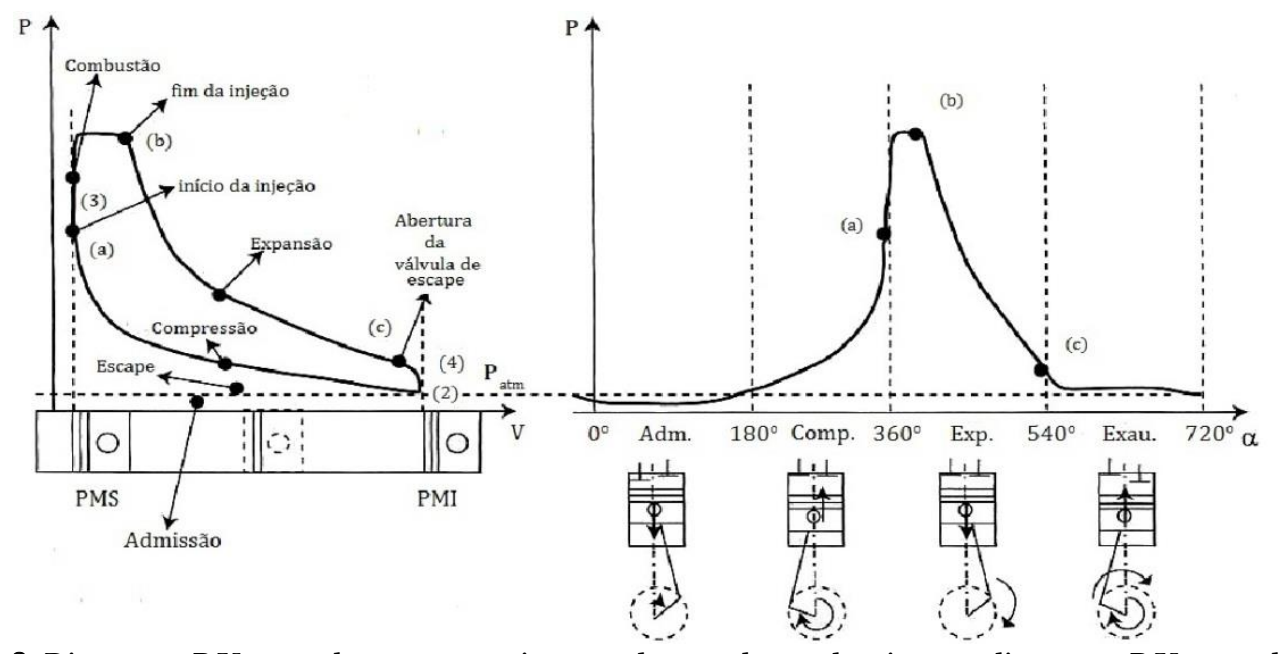

Figura 2. Diagrama P-V em relação ao movimento de translação do pistão e diagrama P-V em relação ao ângulo $(\alpha)$ do virabrequim do motor a combustão por compressão (Brunetti 2012: 86). 


\section{Análise Exergética}

A eficiência energética é uma medida de desempenho de dispositivos à luz da Primeira Lei da Termodinâmica, não levando em consideração o máximo de desempenho possível dos mesmos. Como exemplo, considere duas máquinas térmicas com a mesma eficiência energética (neste caso as duas máquinas convertem a mesma porcentagem do calor fornecido em Trabalho). Considere que a fonte quente, que fornece calor para máquina $1\left(T_{H 1}\right)$, possui temperatura maior comparada com a fonte quente que fornece calor para máquina $2\left(T_{\mathrm{H}_{2}}\right)$, e que ambas compartilham a mesma fonte fria $\left(T_{L}\right)$ (Figura 3). A máxima eficiência que cada máquina pode ter é a mesma da máquina reversível ou máquina de Carnot (Çengel \& Boles 2006) ${ }^{1}$, cuja eficiência é dada por:

${ }^{1} \mathrm{~A}$ Máquina de Carnot é uma máquina ideal que utiliza calor para realizar Trabalho (Çengel \& Boles 2006).

$$
\eta_{\text {rev } 1}=1-\frac{T_{L}}{T_{H 1}}, \quad \eta_{\text {rev } 2}=1-\frac{T_{L}}{T_{H 2}}
$$

Na equação acima, $\eta_{r e v 1}$ é a eficiência da máquina reversível operando com os mesmos níveis de temperatura que a máquina 1 opera, e $\eta_{r e v 2}$ é a eficiência da máquina reversível operando com os mesmos níveis de temperatura que a máquina 2 opera. Como $T_{H 1}>T_{H 2}$, consequentemente, temos que $\eta_{\text {rev } 1}>\eta_{\text {rev2 } 2}$.

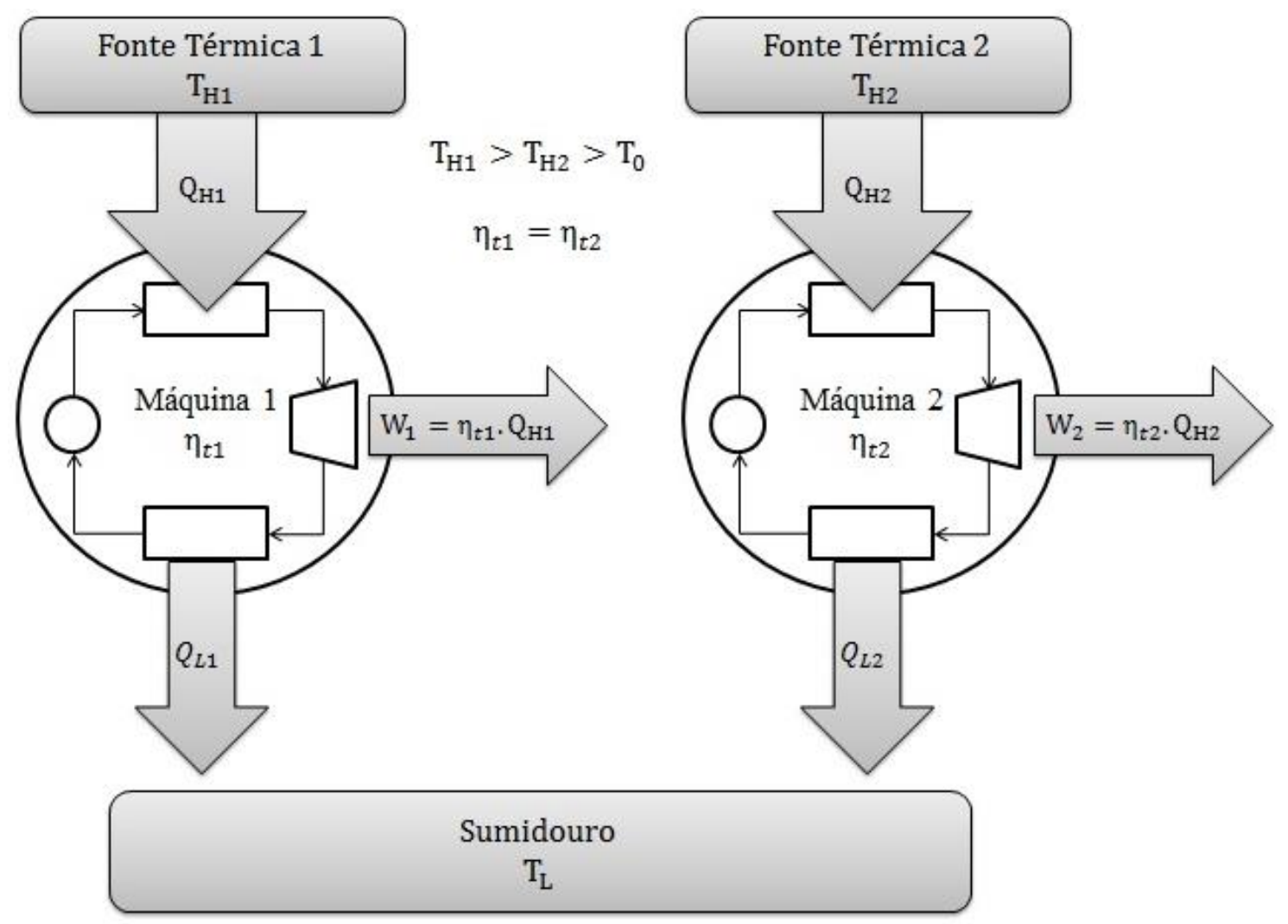

Figura 3. Representação das máquinas térmicas 1 e 2 (Fonte: Dos autores). $Q_{L}$ representa o calor cedido, $Q_{H}$ o calor aportado, e $W_{0}$ Trabalho realizado. 
Embora as duas máquinas tenham o mesmo desempenho à luz da Primeira Lei da Termodinâmica, o mesmo não pode ser dito à luz da Segunda Lei $\left(\eta_{\text {rev } 1}>\right.$ $\left.\eta_{\text {rev2 } 2}\right)^{2}$. Portanto, a máquina 1 apresenta um limite de desempenho maior do que o da máquina 2. Deste modo, pode-se concluir que a máquina 1 possui um desempenho

${ }^{2} \mathrm{~A}$ Primeira Lei da Termodinâmica ! estabelece a conservação da energia em qualquer transformação, enquanto que a Segunda Lei estabelece condições para que as transformações termodinâmicas possam ocorrer.

inferior ao da máquina 2, pois o seu rendimento está mais distante de seu limite superior em comparação à máquina 2. Exemplifica-se, deste modo, que a eficiência à luz da Primeira Lei, não indica de fato o desempenho do dispositivo, e sim somente a capacidade que um dispositivo possui em converter energia. Assim sendo, para superar essa limitação é definida a chamada eficiência exergética $\left(\eta_{I I}\right)$, que consiste na razão entre a eficiência energética $\left(\eta_{t}\right)$, e a máxima eficiência energética possível (eficiência da máquina reversível) sob as mesmas condições (Shapiro et al. 2014),

$$
\eta_{I I}=\frac{\eta_{t}}{\eta_{\text {rev }}}
$$

Ao se calcular a eficiência exergética das duas máquinas, obtém-se:

$$
\frac{\eta_{t 1}}{\eta_{\text {rev } 1}}<\frac{\eta_{t 2}}{\eta_{\text {rev2 }}}
$$

Ou seja, a máquina 1 converte uma porcentagem menor da energia disponível em trabalho útil em comparação à máquina 2. A eficiência exergética também pode ser definida como a razão entre o trabalho útil produzido e o trabalho produzido caso fosse uma máquina reversível (Çengel \& Boles 2006).

\section{Ciclo Dual}

$\mathrm{Na}$ prática, os motores de ignição por centelha, assim como os de ignição por compressão, não possuem um fornecimento de calor totalmente de forma isocórica ou isobárica, respectivamente, o que pode ser visto nas Figuras 1-2. Os motores possuem um aumento de pressão no início da combustão, que pode ser representado por uma isocórica, e logo depois um patamar de pressão, que pode ser representado por uma isobárica (Brunetti 2012: 116).

Nos motores de ignição por centelha operando em altas rotações, o pistão se desloca rapidamente, consequentemente a combustão não acontece em pequenas variações da posição do pistão. Deste modo, o patamar de pressão no diagrama P-V aumenta, pois parte significativa da combustão ocorre enquanto o pistão está se deslocando no sentido do PMS (Ponto Morto Superior) para o PMI (Ponto Morto Inferior). O aumento da pressão proveniente da combustão é anulado pela redução da pressão oriunda do crescimento do volume. Normalmente, os motores de ignição por compressão são modelados pelo Ciclo Diesel (hipótese de fornecimento de calor a pressão constante), porém por razões semelhantes a do motor de ignição por centelha lento, parte da combustão ocorre aproximadamente a volume constante. Portanto, uma modelagem mais adequada considera um pico de pressão a volume constante e um patamar de pressão constante (Figura 4). Essa modelagem é chamada de Ciclo Dual, e tem como casos particulares os Ciclos Otto e Diesel (Ebrahimi 2010). 


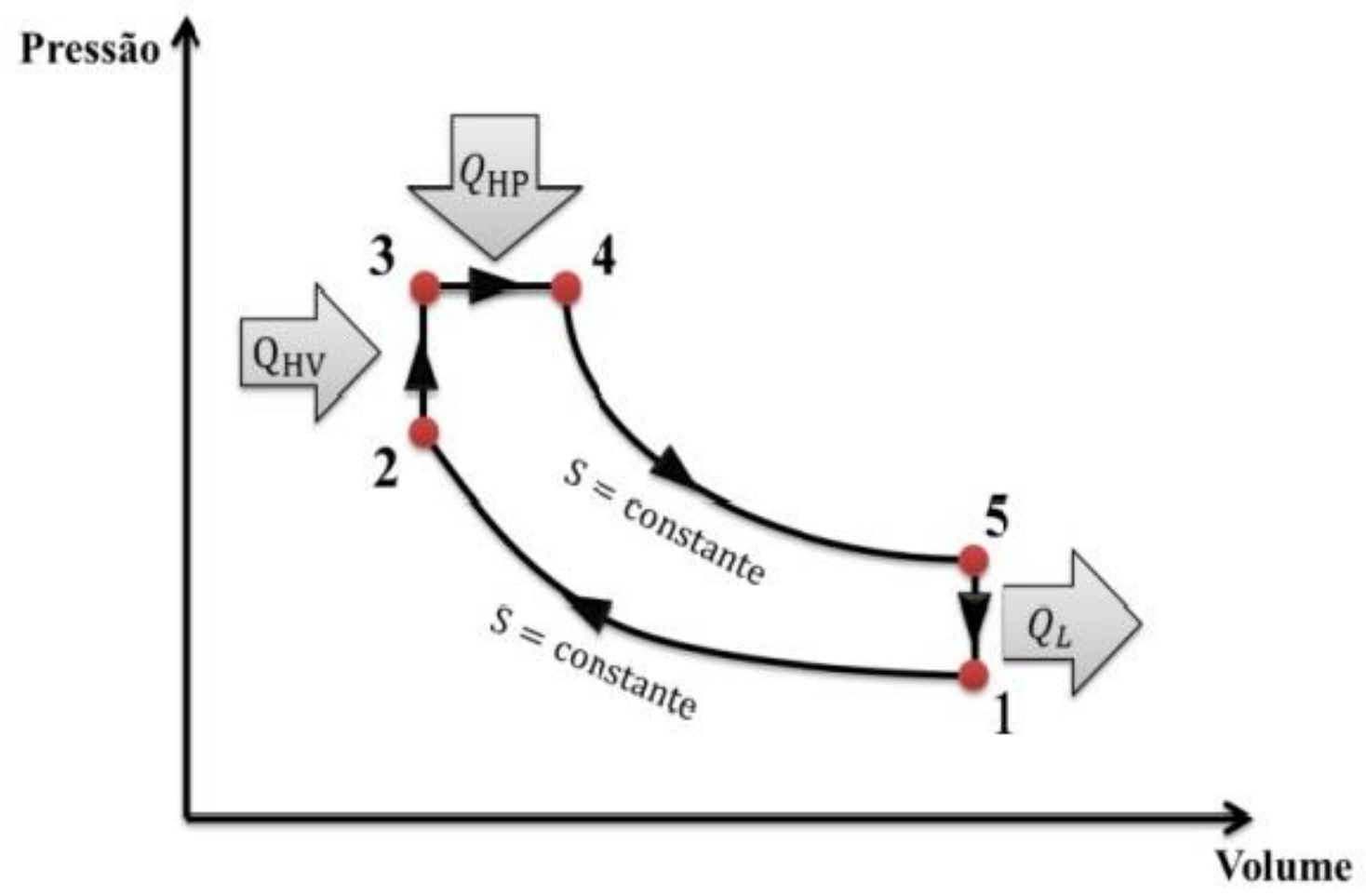

Figura 4. Representação do Diagrama P-V para o Ciclo Dual (Fonte: Dos autores).

Nos motores de combustão interna alternativos, o fluido de trabalho percorre um ciclo termodinâmico aberto, ou seja, ele não retorna para o estado inicial, e sim é exaurido após a explosão, e depois renovado na admissão. Logo, além de calor e trabalho, energia na forma de massa atravessa a fronteira. Esta análise pode ser feita a partir de volumes de controle, porém é usual a utilização da simplificação que consiste em substituir a exaustão e a admissão pela rejeição de calor a volume constante, a fim de torná-lo um sistema fechado. Com o intuito de substituir a reação de combustão que ocorre na mistura ar-combustível dentro da etapa de explosão por um processo mais simples que não envolve reações químicas, considera-se essa etapa como um fornecimento de calor para o fluido de trabalho, que é considerado ar puro simplificado como gás ideal. Em alguns casos, os calores específicos são constantes. As etapas de compressão e explosão, onde ocorrem respectivamente os processos de compressão e expansão do fluido de trabalho, são consideradas isentrópicas, ou seja, onde a entropia do sistema permanece constante. O termo padrão a ar resume o conjunto de hipóteses simplificadoras que preconizam os seguintes pressupostos:

- O fluido de trabalho é ar puro;

- O ar é um gás ideal;

- Não há admissão e nem exaustão. Ao invés disso há uma rejeição de calor a volume constante para retornar ao estado inicial;

- Os processos de compressão e expansão são isentrópicos;

- A combustão é substituida por um fornecimento de calor para o fluido de trabalho;

- Todos os processos são considerados internamente reversíveis; 
- A entalpia (H) e entropia (S) dependem da temperatura $(\mathrm{T})$,
segundo as equações (McBride et al. 2002):

$$
\begin{aligned}
& \frac{H^{0}(T)}{R T}=-a_{1} T^{-2}+a_{2} \frac{\ln (T)}{T}+a_{3}+a_{4} \frac{T}{2}+a_{5} \frac{T^{2}}{3}+a_{6} \frac{T^{3}}{4}+a_{7} \frac{T^{4}}{5}+\frac{b_{1}}{T} \\
& \frac{S^{0}(T)}{R}=-a_{1} \frac{T^{-2}}{2}-a_{2} T^{-1}+a_{3} \ln (T)+a_{4} T+a_{5} \frac{T^{2}}{2}+a_{6} \frac{T^{3}}{3}+a_{7} \frac{T^{4}}{4}+b_{2}
\end{aligned}
$$

onde $R$ é a constante universal dos gases, e $a_{i}^{S}$ são constantes arbitrárias;

- Tais constantes foram ajustadas segundo McBride et al. (2002), e tem como estado de referência a temperatura de $298.15 \mathrm{~K}$ e pressão de 1 bar;

No ciclo, o fluido de trabalho passa pelos seguintes processos ilustrados na Figura 4 (Ebrahimi 2010):

- Processo do estado 1 para o 2: Compressão adiabática (ou seja, sem troca de calor com o ambiente) sem efeitos como o atrito e a expansão não resistida;

- Processo do estado 2 para o 3: Fornecimento de calor isocórico $\left(Q_{H V}\right)$;

- Processo do estado 3 para o 4: Fornecimento de calor isobárico $\left(Q_{H P}\right)$;

- Processo do estado 4 para o 5: Expansão adiabática sem efeitos como o atrito e a expansão não resistida;

- Processo do estado 5 para o 1: Rejeição de calor isocórico $\left(Q_{L}\right)$.

\section{Metodologia}

De modo a obter-se os parâmetros necessários para o cálculo da eficiência exergética desenvolveu-se o estudo paramétrico cujas hipóteses foram apresentadas na seção anterior. Um código em Python ${ }^{\circledR}$ foi desenvolvido para o tratamento de cada um dos diferentes parâmetros de entrada, a partir de seus valores iniciais, até o final da análise em um passo determinado pelo usuário. Assim, em cada análise, um parâmetro de entrada escolhido irá receber um acréscimo, enquanto os outros parâmetros permanecem constantes, e assim por diante. Em cada iteração, o valor assumido de cada parâmetro presente na análise é guardado em uma tabela.

O procedimento para solução dos parâmetros em cada processo é descrito abaixo:

- Parâmetros de entrada:

O calor total aportado $\left(Q_{H}\right)$ é a soma das etapas isocórica $\left(Q_{H V}\right)$ e isobárica $\left(Q_{H P}\right)$ :

$$
Q_{H}=Q_{H V}+Q_{H P}
$$

O calor aportado de forma isocórica $\left(Q_{H V}\right)$ é proporcional a $(\phi)$ 


$$
Q_{H V}=\phi Q_{H}
$$

Onde $\phi$ é o percentual de calor aportado de forma isocórica. Combinando (6) e (7) temos o calor aportado de forma isobárica $\left(Q_{H P}\right)$ :

$$
Q_{H P}=(1-\phi) Q_{H}
$$

Considerando-se que são conhecidos os valores para $T_{1}, P_{1}$ e $r$, respectivamente, temperatura do estado 1, pressão do estado 1 e taxa de compressão, pode-se analisar o processo do estado 1 para o 2.

- Processo do estado 1 para o 2 (compressão isentrópica):

Uma vez que tem-se como entrada a temperatura $\left(T_{1}\right)$ e pressão $\left(P_{1}\right)$, do estado 1 , é possível calcular-se o volume $\left(V_{1}\right)$ e a entropia $\left(S_{1}\right)$, do estado 1 , usando as seguintes equações (Çengel \& Boles 2006):

$$
\begin{gathered}
S_{1}=S^{0}\left(T_{1}\right)-R \ln \left(\frac{P_{1}}{P_{r e f}}\right) \\
P_{1} V_{1}=R T_{1} \\
U_{1}=H^{0}\left(T_{1}\right)-R T_{1}
\end{gathered}
$$

Nas equações acima, os índices denotam o estado. $P_{r e f}$ é a pressão de referência (McBride et al. 2002), e $U_{1}$ é a função de estado energia interna, que é definida por $U=H-P V$.

Em (9) $S^{0}$ é dada pela equação (5). A equação leva em conta a variação da entropia em relação a temperatura. Assim, se faz necessário adicionar o termo $\left(-\mathrm{R} \ln \left(P_{1} / P_{\text {ref }}\right)\right)$, devido a variação da entropia do estado de referência até o estado em questão (já que o mesmo encontrase em outra pressão, i.e. $S_{1}$ ) (Çengel \& Boles 2006).

As equações (10) e (11) são aplicações diretas das definições de energia interna, entalpia e do modelo de gás ideal.

Do estado 2 conhecemos a entropia pois o processo é isentrópico (i.e. $S_{2}=S_{1}$ ). Sabemos ainda que o volume é função da taxa de compressão ( $r$ (Ebrahimi 2010). Assim,

$$
\begin{gathered}
V_{2}=\frac{V_{1}}{r} \\
S_{2}\left(T_{2}\right)-S_{1}=0
\end{gathered}
$$

Para se obter $T_{2}$ basta encontrar-se a raiz de (13). Na implementação (linhas 116 a 122 do Apêndice A) este valor é encontrado através da função newton da biblioteca scipy.optimize. Conhecida a temperatura $T_{2}$ e as equações (10) e (11) é possível obter-se $U_{2}$ e $P_{2}$.

- Processo do estado 2 para o 3 (fornecimento de calor a volume constante): 
O vínculo dos estados 2 e 3 é o processo de adição de calor a volume constante. Então, da definição de energia interna vem a relação:

$$
U_{3}\left(T_{3}\right)=U_{2}+Q_{H V} \rightarrow U_{3}\left(T_{3}\right)-U_{2}-Q_{H V}=0
$$

Para se determinar $T_{3}$ basta encontrar a raiz de (14), obtida na implementação através da função hinv, com base nas definições de energia interna e entalpia e da equação (10). A raiz novamente é obtida através da função newton da biblioteca scipy.optimize.

Com o valor de $T_{3}$ e as equações (4), (5), (9) e (10) é possível obter-se $H_{3}, S_{3}^{0}, P_{3}$ e $S_{3}$, respectivamente.

- Processo do estado 3 para o 4 (fornecimento de calor a pressão constante):

A ligação dos estados 3 e 4 é o processo de adição de calor a pressão constante. Então, da definição de entalpia vem a relação:

$$
H_{4}\left(T_{4}\right)=H_{3}+Q_{H P} \rightarrow H_{4}\left(T_{4}\right)-H_{3}-Q_{H P}=0
$$

O processo de obtenção de $T_{4}$ é o mesmo descrito na etapa dos processos 2 para 3.

Com o valor de $T_{4}$ e as equações (4), (5), (9-11) é possível obter-se $H_{4}, S_{4}^{0}, P_{4}, S_{4}$ e $U_{4}$, respectivamente.

- Processo do estado 4 para o 5 (expansão isentrópica):

A correlação entre os estados 4 e 5 é a mesma dos estados 1 e 2 pelas hipóteses, $S_{5}=S_{4}$ e $V_{5}=V_{1}$. Então, o processo de solução de $T_{5}$ procede da mesma forma que o de $T_{2}$. Com o valor de $T_{5}$ tem-se $U_{5}$ e $P_{5}$, como visto no estado 2 .

- Processo do estado 5 para o 1 (rejeição de calor a volume constante):

Nesse ponto todas as variáveis termodinâmicas relevantes para as análises já foram levantadas, e o encadeamento é o mesmo dos pontos 2 e 3 . Assim, aplicar as equações nesse passo tem efeito de prova real.

\section{Estudo Paramétrico}

Tomando-se como base o calor fornecido na combustão $\left(Q_{H}=700 \frac{\mathrm{kj}}{\mathrm{kg}}\right)$ e a condição do estado morto $\left(T_{\mathrm{H}}=2600 \mathrm{~K}, T_{0}=298.15 \mathrm{~K}\right.$ e $\left.P_{0}=100 \mathrm{kPa}\right)$, e aplicando-se uma série de estudos paramétricos para diversas taxas de compressão $(r)$ e percentuais de calor aportado de forma isocórica $(\phi)$, tem-se os resultados apresentados na Tabela 1 , onde considera-se as colunas na seguinte ordem: fração do calor fornecido a volume constante $\left(C_{\text {volcte }}\right)$, eficiências energética $\left(E_{\text {energ }}\right)$ e exergética $\left(E_{\text {exerg }}\right)$, exergia destruída no fornecimento de calor isocórico $\left(\boldsymbol{x}_{\text {des23 }}\right)$, exergia destruída no fornecimento de calor isobárico $\left(x_{\text {des } 34}\right)$, exergia destruída total no fornecimento de calor $\left(\boldsymbol{x}_{\text {descalor }}\right)$, exergia destruída na rejeição de calor isocórico $\left(\boldsymbol{x}_{\text {des51 }}\right)$, exergia destruída total no ciclo $\left(x_{\text {destotal }}\right)$, razão entre a exergia destruída total e a exergia fornecida $(\lambda)$.

A partir do estudo paramétrico, é possível identificar as variáveis mais impactantes no sentido de diminuir a destruição de exergia (Bahlouli et al. 2015). Observa-se na Tabela 1, que quando $\phi=1$ tem-se o aumento da taxa de compressão $(r)$ e do percentual de calor aportado de forma isocórica. Nota-se que o processo onde há maior destruição de exergia é o de rejeição de calor de forma isocórica (ver coluna $\boldsymbol{x}_{\text {destotal }}$ ), o que serve de indicativo para possíveis melhorias do dispositivo/forma de operação que acarretem em diminuição na destruição de exergia a fim de levar a máquina térmica à maior eficiência possível. 
Tabela 1. Estudos paramétricos para diferentes taxas de compressão.

\begin{tabular}{clcccccccc}
\hline $\mathbf{r}$ & $\boldsymbol{C}_{\text {volcte }}$ & $\boldsymbol{E}_{\text {energ }}$ & $\boldsymbol{E}_{\text {exerg }}$ & $\boldsymbol{x}_{\text {des23 }}$ & $\boldsymbol{x}_{\text {des34 }}$ & $\boldsymbol{x}_{\text {descalor }}$ & $\boldsymbol{x}_{\text {des51 }}$ & $\boldsymbol{x}_{\text {destotal }}$ & $\boldsymbol{\lambda}$ \\
\hline \multirow{4}{*}{5} & $\phi=0.0$ & 0.3476 & 0.3926 & 0.0 & 166.3383 & 166.3383 & 210.0561 & 376.3944 & 0.6074 \\
& $\phi=0.25$ & 0.4015 & 0.4535 & 57.6466 & 95.8093 & 153.4559 & 185.2498 & 338.7057 & 0.5465 \\
& $\phi=0.5$ & 0.4260 & 0.4812 & 96.194 & 51.1583 & 147.3523 & 174.1745 & 321.5267 & 0.5188 \\
& $\phi=0.75$ & 0.4366 & 0.4932 & 123.7101 & 20.9519 & 144.662 & 169.4281 & 314.0901 & 0.5068 \\
& $\phi=1.0$ & 0.4394 & 0.4963 & 143.9475 & 0.0 & 143.9475 & 168.1813 & 312.1288 & 0.5037 \\
\hline \multirow{4}{*}{10} & $\phi=0.0$ & 0.5057 & 0.5712 & 0.0 & 126.3919 & 126.3919 & 139.3224 & 265.7142 & 0.4288 \\
& $\phi=0.25$ & 0.5365 & 0.6060 & 42.564 & 75.2327 & 117.7967 & 126.4024 & 244.1992 & 0.3940 \\
& $\phi=0.5$ & 0.5518 & 0.6233 & 72.5559 & 40.8214 & 113.3773 & 120.0585 & 233.4358 & 0.3767 \\
& $\phi=0.75$ & 0.5589 & 0.6313 & 94.4907 & 16.8337 & 111.3244 & 117.1798 & 228.5042 & 0.3687 \\
& $\phi=1.0$ & 0.5608 & 0.6335 & 110.7609 & 0.0 & 110.7609 & 116.3971 & 227.158 & 0.3665 \\
\hline \multirow{4}{*}{15} & $\phi=0.0$ & 0.5773 & 0.6521 & 0.0 & 105.8804 & 105.8804 & 109.7517 & 215.632 & 0.3479 \\
& $\phi=0.25$ & 0.5995 & 0.6771 & 35.2193 & 63.9103 & 99.1296 & 100.95 & 200.0796 & 0.3229 \\
& $\phi=0.5$ & 0.6112 & 0.6903 & 60.6154 & 34.89 & 95.5054 & 96.4085 & 191.9139 & 0.3097 \\
& $\phi=0.75$ & 0.6167 & 0.6966 & 79.3691 & 14.4044 & 93.7735 & 94.2829 & 188.0564 & 0.3034 \\
& $\phi=1.0$ & 0.6182 & 0.6983 & 93.2894 & 0.0 & 93.2894 & 93.6939 & 186.9833 & 0.3017 \\
\hline \multirow{4}{*}{20} & $\phi=0.0$ & 0.6206 & 0.7010 & 0.0 & 92.5224 & 92.5224 & 92.7652 & 185.2876 & 0.299 \\
& $\phi=0.25$ & 0.6383 & 0.7210 & 30.5818 & 56.2553 & 86.8371 & 86.0554 & 172.8926 & 0.279 \\
& $\phi=0.5$ & 0.6479 & 0.7319 & 52.9154 & 30.7828 & 83.6983 & 82.4806 & 166.1789 & 0.2681 \\
& $\phi=0.75$ & 0.6526 & 0.7371 & 69.4757 & 12.6946 & 82.1702 & 80.7733 & 162.9435 & 0.2629 \\
& $\phi=1.0$ & 0.6539 & 0.7385 & 81.7378 & 0.0 & 81.7378 & 80.294 & 162.0318 & 0.2615 \\
\hline
\end{tabular}

\section{Conclusões}

De acordo com os resultados é possível afirmar que a eficiência do Ciclo Dual aumenta com o crescimento da adição de calor a volume constante (mantendo fixo o calor total fornecido). Diante disso, para os motores alternativos de combustão interna é vantajoso em termos de ganho das eficiências energética e exergética, que a combust ao seja realizada a volume constante. Os resultados indicam que o crescimento da taxa de compressão resulta no aumento das eficiências energética e exergética do Ciclo Dual, entretanto também apresentam o efeito de tornar o crescimento das mesmas, em relação ao aumento do fornecimento de calor a volume constante (mantendo fixo o calor total fornecido), menos acentuado. Logo, para os motores de ignição por compressão, que operam em altas taxas de compressão, aumentar a combustão realizada a volume constante, por motivos de ordem prática, não é sempre vantajoso. Para trabalhos futuros, pretende-se realizar um estudo de caso para motores de combustão interna, considerando o levantamento do custo agregado da destruição de exergia (análise termo-econômica). Além disso, pretende-se analisar as possibilidades de aproveitamento da exergia do calor rejeitado para outros processos em motor estacionário. Outra meta é realizar uma análise comparativa teórico-experimental, tendo como base os resultados levantados no presente trabalho. Finalmente, pretende-se investigar possibilidades de melhorias no processo, analisando onde ocorre a maior destruição de exergia em um estudo de caso aplicado à área de motores alternativos de combustão interna.

\section{Agradecimentos}

Aos avaliadores anônimos pela revisão crítica do manuscrito. O presente trabalho foi realizado com apoio da Coordenação de Aperfeiçoamento de Pessoal de Nível Superior - Brasil (CAPES).

\section{Referências}

Bahlouli K., Saray R.K. \& Sarabchi N. (2015) Parametric investigation and thermo-economic multi-objective optimization of an ammonia - water power/cooling cycle coupled with an 
HCCI (homogeneous charge compression ignition) engine. Energy, 86: 672-684. Doi: 10.1016/j.energy.2015.04.022

Brunetti F. (2012) Motores de Combustão Interna. Volume 2. $3^{\circ}$ edição. São Paulo: Blucher. 486 p. Çengel Y.A. \& Boles M.A. (2006) Termodinâmica. $5^{\circ}$ edição. São Paulo: Mc-GrawHill. 740 p.

Ebrahimi R. (2010) Performance Analysis of a Dual Cycle Engine with Considerations of Pressure Ratio and Cut-Off Ratio. Acta Physica Polonica A, 118(4): 534-539.

Heywood J.B. (2011) Internal combustion engine fundamentals. Delhi: Mc-GrawHill. 930 p.

McBride B.J., Zehe M.J. \& Gordon S. (2002) NASA Glenn Coefficients for Calculating Thermodynamic Properties of Individual Species. NASA/TP—2002-211556.

Shapiro H.N., Moran M.J., Boettner D.D. \& Bailey M.B. (2014) Principles of Engineering Thermodynamics. $7^{\circ}$ edition. Delhi: Wiley. 855 p. 


\section{Apêndice A - Implementação}

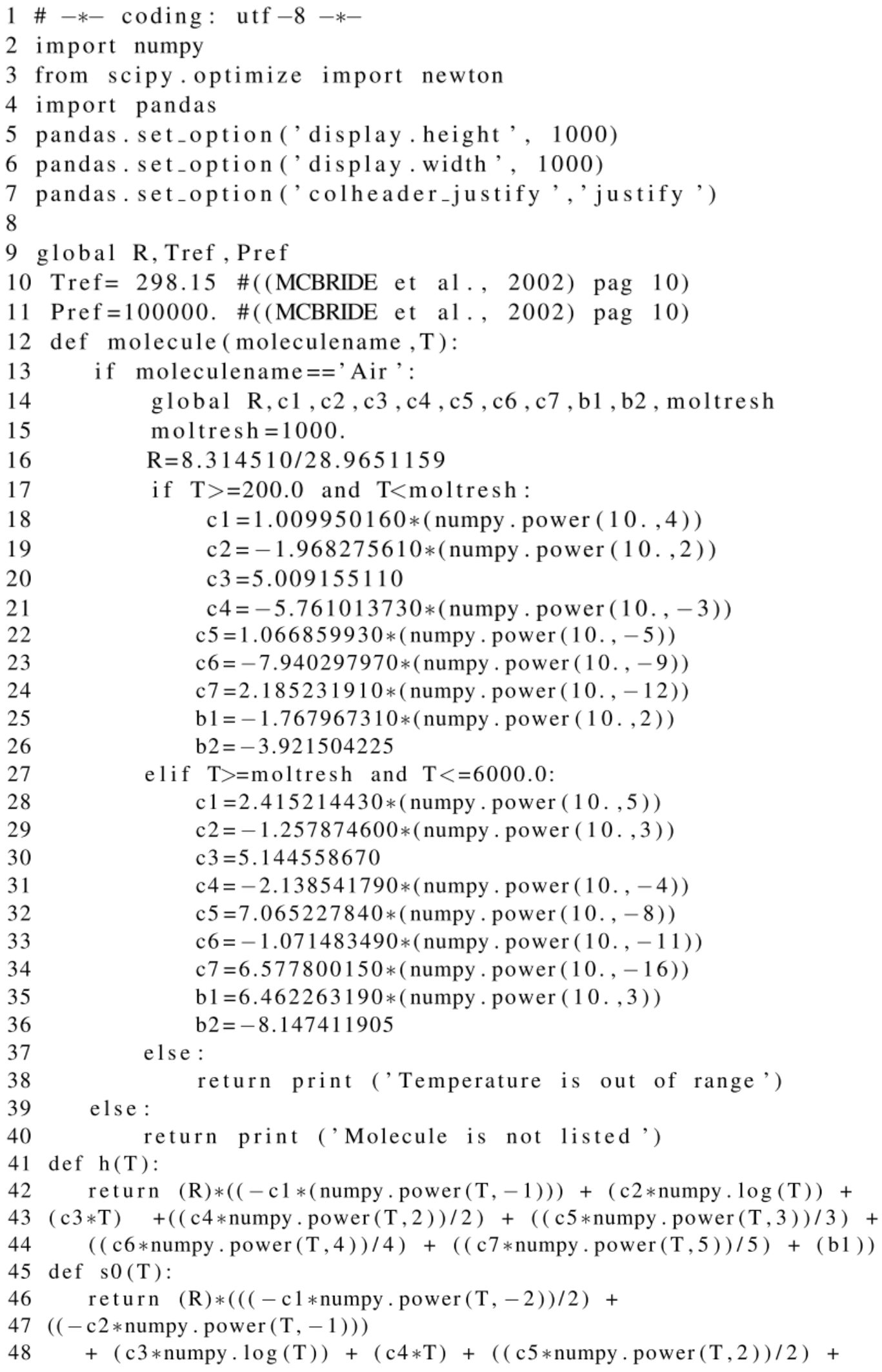




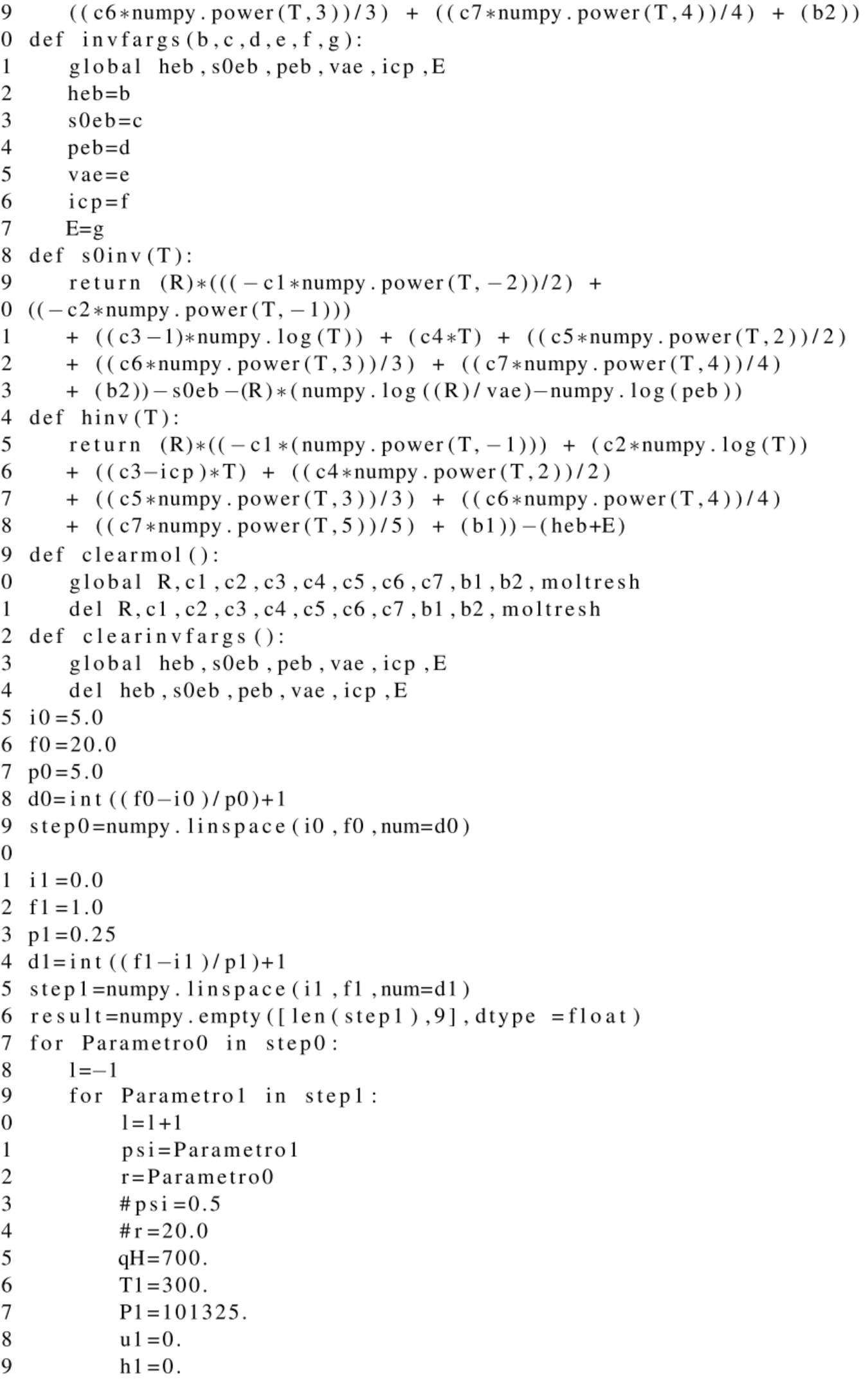




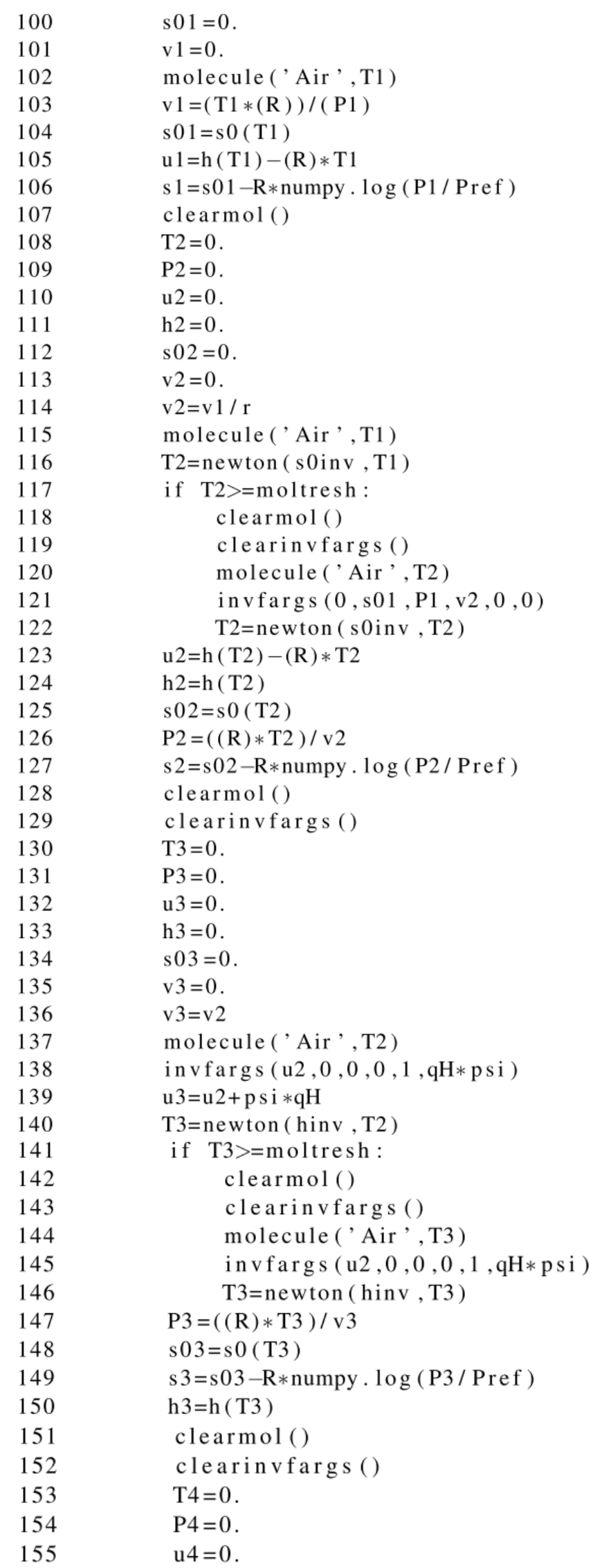




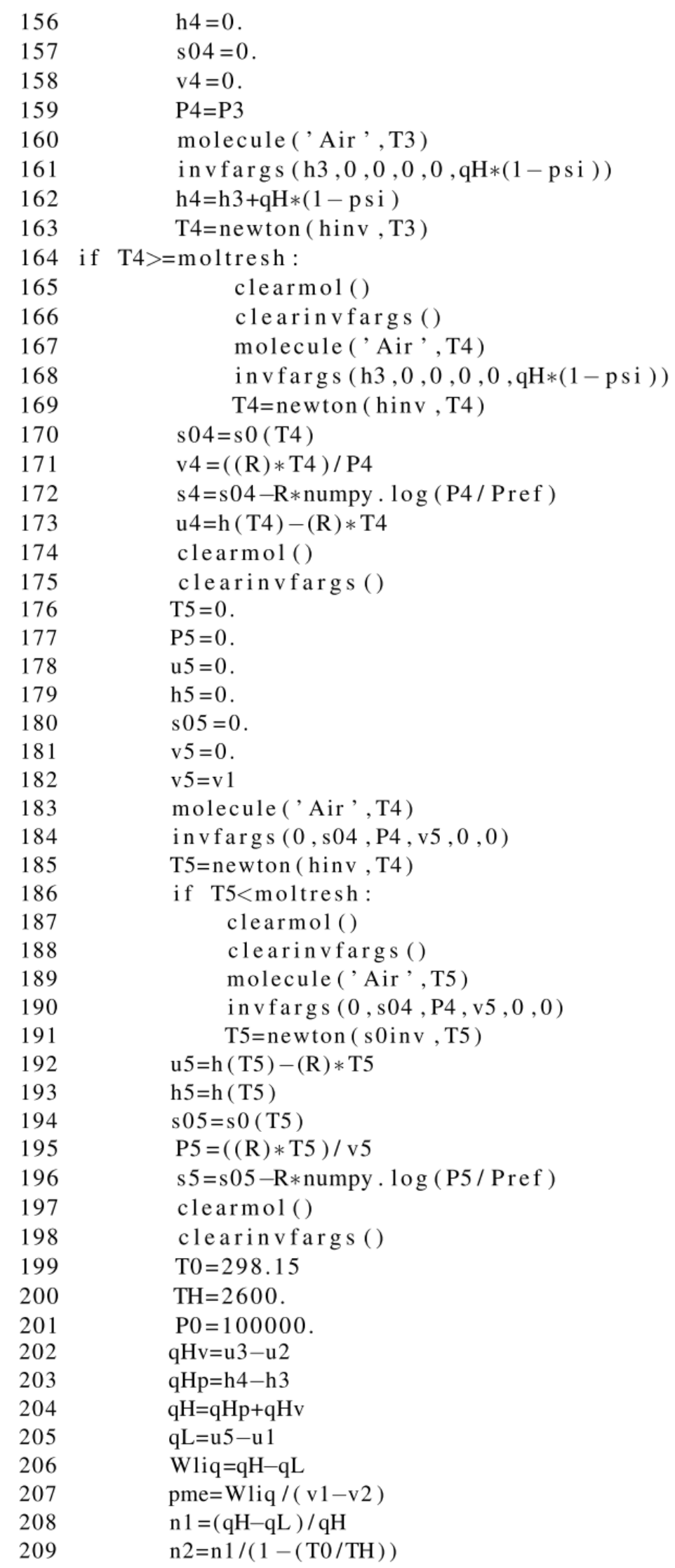




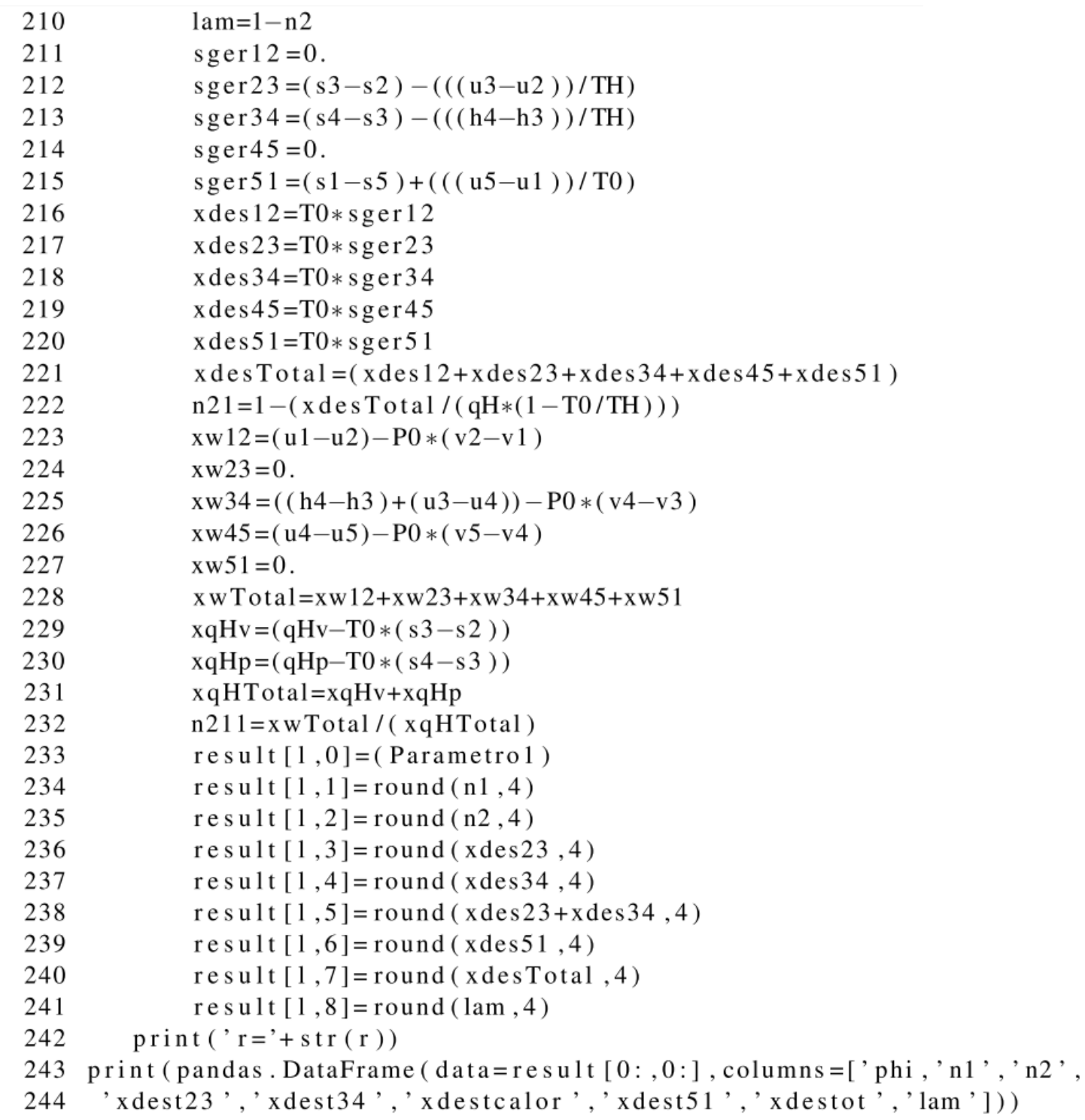

